\title{
Liraglutide reduces hepatic glucolipotoxicity-induced liver cell apoptosis through NRF2 signaling in Zucker diabetic fatty rats
}

\author{
JUN GUO $^{1 *}, \mathrm{CAI} \mathrm{LI}^{1,2^{*}}$, CHUNXIAO YANG ${ }^{1,3}$, BING LI $^{4}, \mathrm{JIE} \mathrm{WEI}^{1}$, \\ YAJUN LIN ${ }^{1}$, PENG YE ${ }^{1}$, GANG HU$^{1}$ and JIAN LI ${ }^{1}$ \\ ${ }^{1}$ The MOH Key Laboratory of Geriatrics, Beijing Hospital, National Center of Gerontology, Beijing 100730; \\ ${ }^{2}$ Department of Internal Medicine, The Affiliated Hospital of Qingdao University, Qingdao, Shandong 266071; \\ ${ }^{3}$ Peking University Fifth School of Clinical Medicine, Beijing Hospital, Beijing 100730; ${ }^{4}$ Department of \\ Encephalopathy, Medical Department of Chinese Medicine, Shandong University of \\ Traditional Chinese Medicine, Jinan, Shandong 250355, P.R. China
}

Received December 19, 2017; Accepted April 17, 2018

DOI: $10.3892 / \mathrm{mmr} .2018 .8919$

\begin{abstract}
The primary aim of the present study was to evaluate the effects of liraglutide on glucolipotoxicity-induced liver cell apoptosis and the underlying mechanisms in Zucker diabetic fatty $(\mathrm{ZDF})$ rats. The results revealed that liraglutide significantly decreased the body weight, hyperglycemia and hyperlipidemia of ZDF rats relative to those of Zucker lean $(\mathrm{ZL})$ rats $(\mathrm{P}<0.05)$. Furthermore, the reduced liver cell apoptosis was observed in the ZDF rats following 6 weeks of liraglutide therapy. These data validated the beneficial effects of liraglutide on diabetic and obese ZDF rats. In addition, novel data was obtained that demonstrated that liraglutide treatment increased the expression of the antioxidant transcription factor nuclear factor-erythroid 2-related factor 2 (NRF2), as well as the transcription of downstream target genes, including nicotinamide adenine dinucleotide phosphate quinone dehydrogenase 1 and heme oxygenase-1 $(\mathrm{P}<0.05)$. Additionally, serum and hepatic GSH and SOD levels increased following liraglutide therapy $(\mathrm{P}<0.05)$. Hence, it was proposed that liraglutide may enhance the antioxidant activity of liver cells by activating the NRF2 signaling pathway, thereby reducing liver cell apoptosis induced by glucolipotoxicity in ZDF rats, which may shed light on the application of liraglutide in the treatment of diabetes- and obesity-induced liver injury.
\end{abstract}

Correspondence to: Dr Gang Hu or Dr Jian Li, The MOH Key Laboratory of Geriatrics, Beijing Hospital, National Center of Gerontology, 1 Dahua Road, Beijing 100730, P.R. China

E-mail: hu61@hotmail.com

E-mail: lijian@bjhmoh.cn

*Contributed equally

Key words: liraglutide, glucolipotoxicity, liver cell apoptosis, nuclear factor-erythroid 2-related factor 2 signaling

\section{Introduction}

At present, energy surplus has become the main factor endangering human health (1). Nonalcoholic fatty liver (NAFLD) and type 2 diabetes (T2D) are metabolic diseases that are closely related to energy surplus (1). With the increasing acceleration of economic development, metabolism-related diseases, which are characterized by excess energy, are increasing year by year (2). Statistical analysis showed that in 2010, the prevalence rate of diabetes in China was as high as $11.6 \%(3,4)$. At the same time, the incidence of NAFLD reached $20.9 \%$ in China, and the number of current NAFLD patients is more than 200 million (2). Therefore, NAFLD and type 2 diabetes (T2D) have become major obstacles to the health of people around the world.

The liver is the major organ that promotes the synthesis and degradation/oxidation of fatty acids (FAs), as well as the metabolism of cholesterol and phospholipids. In hepatocytes, excess dietary glucose can be converted into fat, which can be then stored as triglycerides (TGs) in lipid droplets (5). Once the fatty acid input exceeds the capacity of $\beta$-oxidation, the accumulated acyl-CoA is drained by triglyceride synthesis, and the oxidation of FFAs is increased (6). Enhanced FFA $\beta$-oxidation leads to higher levels of free radical formation and more hydrogen peroxide production in peroxisomes, thereby causing the production of reactive oxygen species (ROS) $(7,8)$.

Multiple genes are suggested to be involved in ROS production and glucolipotoxicity $(9,10)$. Among these genes, nuclear factor-erythroid 2-related factor 2 (Nfe212/NRF2) is a basic leucine zipper transcription factor that regulates the expression of detoxifying and antioxidant genes, such as heme oxygenase 1 (HO-1), superoxide dismutase (SOD), and catalase (CAT) $(9,10)$. Under normal conditions, NRF2 is mainly localized in the cytoplasm via interactions with Kelch-like ECH-associated protein 1 (Keap1). In response to oxidative stress, NRF2 translocates into the nucleus and then initiates the expression of antioxidant enzymes through binding antioxidant response element (ARE) (11-13). Thus, NRF2 plays a key role in the antioxidant and cytoprotective defense system in the liver. 
Glucagon-like peptide-1 (GLP-1) receptor agonists (GLP-1RAs) belong to a novel class of antidiabetic medications that regulate glucose homeostasis mainly by interacting with GLP-1 receptors $(14,15)$. Liraglutide is a synthetic GLP-1RA that has high structural similarity to human GLP-1 (16). Recent studies have shown that liraglutide is characterized by anti-inflammatory and antioxidant features $(17,18)$. However, whether liraglutide improves glucolipotoxicity-induced liver cell apoptosis is unclear. The primary aim of the current study was to evaluate the effects of liraglutide on glucolipotoxicity-induced liver cell apoptosis and the underlying mechanisms in Zucker diabetic fatty (ZDF) rats.

\section{Materials and methods}

Animals and treatments. Male ZDF rats and Zucker lean (ZL) littermates were purchased at 5 weeks of age from Vital River Laboratory Animal Technology Co. Ltd. (Beijing, China). The animals were housed under controlled temperature $\left(21 \pm 2^{\circ} \mathrm{C}\right)$, relative humidity $(50 \pm 10 \%)$ and artificial light $(12 \mathrm{~h}$ light/dark cycle, lights on at 7 A.M.) conditions. Littermates from the same mother or a foster mother were housed in one large cage with ad libitum access to distilled water and a standard rat diet for ZL rats or a high-fat diet (Purina5008; LabDiet, St. Louis, MO, USA) for ZDF rats except on the days when the rats were fasted for $6 \mathrm{~h}$ and a blood glucose level test was performed. The experimental procedure began when the rats were 8 weeks old. The ZDF rats were further randomly divided into subgroups, including a liraglutide (Novo Nordisk A/S, Bagsvaerd, Denmark) group and a saline group ( $n=8$ for each group). Each rat was given liraglutide $(150 \mathrm{ml} / \mathrm{kg}$ body weight) or saline for 6 weeks. The body weight and fasting blood glucose level were measured every week.

All rat procedures were approved by the Animal Ethics Committee at the MOH Key Laboratory of Geriatrics, Beijing Hospital (BJHMOH-2015-1002).

Biochemical Analysis. The serum biochemical profiles, such as aspartate aminotransferase (AST) and alanine aminotransferase (ALT), were evaluated with a Biochem-Immuno Autoanalyzer (TBA-40FR; Toshiba, Tokyo, Japan).

TUNEL staining. Nuclear fragmentation was evaluated using TUNEL staining with an apoptosis detection kit (R\&D Systems, Inc., Minneapolis, MN, USA) according to the manufacturer's protocol. The total number of apoptotic cells were counted in randomly acquired 10 nonoverlapping high-magnification imaging fields (x40) in each section and an average of apoptotic cell proportion was calculated.

Western blot analysis. Liver tissue samples were extracted using RIPA buffer (Beijing Solarbio Science \& Technology Co., Ltd., Beijing, China). A bicinchoninic protein assay kit (Pierce; Thermo Fisher Scientific, Inc., Waltham, MA, USA) was used to determine the protein concentration. Equal amounts (20 $\mu \mathrm{g}$ each) of proteins were separated by $10 \%$ SDS-PAGE, transferred to PVDF membranes (Merck KGaA, Darmstadt, Germany), blocked with $8 \%$ nonfat dry milk, and then incubated with specific primary antibodies at $4^{\circ} \mathrm{C}$ overnight. Antibodies against NRF2 (ab137550;
Abcam, Cambridge, UK), NAD(P)H quinone dehydrogenase 1 (NQO1) (ab28947; Abcam, Cambridge, UK), heme oxygenase-1 (HO-1) (ab13248; Abcam), cleaved caspase 3 (cat. no. 9664; Cell Signaling Technology, Inc., Danvers, MA, USA), and $\beta$-actin (cat. no. 3700; Cell Signaling Technology, Inc.) were used. Nonspecific binding was blocked using $8 \%$ (w/v) milk in Tris-buffered saline with $1 \%$ Tween-20 (TBST; Beijing Solarbio Science \& Technology Co., Ltd.) for $2 \mathrm{~h}$ at room temperature. Following three washes with TBST (5 min/wash), the membranes were incubated with horseradish peroxidase (HRP)-conjugated goat anti-rabbit and anti-mouse IgG or HRP-conjugated mouse anti-goat IgG (all 1:5,000; Zhongshan Gold Bridge Biological Technology Co., Beijing, China) for $2 \mathrm{~h}$ at room temperature and then washed. $\beta$-actin was used as the internal control. Signals were detected with enhanced chemiluminescence according to the manufacturer's instructions (EMD Millipore, Billerica, MA, USA). ImageJ 2.0 software (National Institutes of Health, Bethesda, MD, USA) was used for density analysis.

Glycogen and triglyceride measurement. The content of tissue glycogen and TGs was measured using a Glycogen Assay kit (ab65620; Abcam) and Triglyceride Assay Quantification kit (ab65336; Abcam).

Hematoxylin and eosin $(H \& E)$ staining. Frozen sections of liver specimens were fixed in paraformaldehyde (Beijing Solarbio Science \& Technology Co., Ltd.) for H\&E staining. In brief, liver tissues were fixed in $4 \%$ paraformaldehyde buffer for $1 \mathrm{~h}$ at $37^{\circ} \mathrm{C}$. Then, the tissues were embedded in optimal cutting temperature (OCT) solution (Sakura Finetek, Tokyo, Japan) on dry ice and cut into $5 \mu \mathrm{m}$ sections. Then, the slides were first incubated with hematoxylin (Beijing Solarbio Science \& Technology Co., Ltd.) for $5 \mathrm{~min}$ and then washed with $1 \%$ ethanol hydrochloride for $3 \mathrm{sec}$. After washing with water, the slides were stained with eosin (Beijing Solarbio Science \& Technology Co., Ltd.) for $3 \mathrm{~min}$ and dehydrated with an alcohol gradient. The vacuoles were considered the lipid droplets (19) and examined under a light microscope (Olympus BH-2; Olympus Corporation, Tokyo, Japan) in a blinded manner by a pathologist.

Periodic acid schiff (PAS) staining. The sections were then stained with PAS reagent (Beijing Solarbio Science \& Technology Co., Ltd.) at room temperature for $2 \mathrm{~h}$ and then washed with $1 \%$ ethanol hydrochloride for $3 \mathrm{sec}$. After washing with water, the slides were stained with eosin (Beijing Solarbio Science \& Technology Co., Ltd.) for $3 \mathrm{~min}$ and dehydrated with an alcohol gradient. Images were evaluated using light microscopy (Olympus BH-2; Olympus Corporation).

Immunohistochemistry (IHC). Liver tissues were fixed with $15 \%$ formalin ( $\mathrm{pH} 7.4$ ), embedded in paraffin, cut into $2 \mu \mathrm{m}$ sections and mounted on slides. IHC staining for cleaved caspase 3 (c-caspase 3) was performed using Histofine Simple Stain MAX-PO MULTI (Nichirei Biosciences Inc., Tokyo, Japan). After deparaffinization with xylene, sections were incubated with $0.3 \%$ hydrogen peroxide for $15 \mathrm{~min}$ to block endogenous peroxidases prior to c-caspase 3 evaluation. For antigen retrieval, sections were incubated for $30 \mathrm{~min}$ 
in $0.01 \mathrm{~mol} / 1$ citrate buffer $(\mathrm{pH} 6.0)$ at $100^{\circ} \mathrm{C}$. Proteinase $\mathrm{K}$ (P9460, Beijing Solarbio Science \& Technology Co., Ltd.) was used for antigen retrieval with incubation for $10 \mathrm{~min}$. After blocking with $10 \%$ goat serum, sections were incubated overnight at $4^{\circ} \mathrm{C}$ with primary antibody against c-caspase 3 at 1:200 dilution (cat. no. 9664, Cell Signaling Technology, Inc.). After washing sections and incubating them with secondary antibody for $1 \mathrm{~h}$ at room temperature, DAB substrate (ZLI-9019; Zhongshan Golden Bridge Biological Technology Co., Beijing, China) was used to visualize the IHC staining. Images were evaluated using light microscopy (Olympus BH-2; Olympus Corporation) in a blinded manner by two pathologists.

Determination of oxidative stress. Blood was collected from the rat hearts in anticoagulation tubes containing EDTA. The liver tissues were homogenized. Then, the blood and homogenates were centrifuged at 1,500 x g for $20 \mathrm{~min}$. The supernatants were collected and used for the determination of antioxidant enzymes using a SOD kit (batch no.: 20130410; Nanjing Jiancheng Bioengineering Institute, Nanjing, China); a malondialdehyde (MDA) kit (batch no.: 20130409; Nanjing Jiancheng Bioengineering Institute); and a glutathione peroxidase (GSH-PX) kit (batch no.: 201304010; Nanjing Jiancheng Bioengineering Institute) according to the instructions.

Statistical analysis. The data are presented as the mean \pm standard error. Analysis was performed with GraphPad v7 software (GraphPad, Inc., La Jolla, CA, USA). Two-tailed unpaired Student's t-tests were used for comparisons between two groups. Analysis of variance was performed for multiple comparison tests followed by Tukey's post hoc test was used for comparisons of two or more groups. $\mathrm{P}<0.05$ was considered to indicate a statistically significant difference.

\section{Results}

Liraglutide improves metabolic disorders in ZDF rats. First, we evaluated the body weight of ZL rats and ZDF rats. Compared with $Z \mathrm{~L}$ rats $(311.5 \pm 9.41,326 \pm 8.05,328.71 \pm 9.97,335.1 \pm 11.38$, $340.86 \pm 13.76,336.71 \pm 8.75 \mathrm{~g}), \mathrm{ZDF}$ rats had significantly higher body weight $(362.7 \pm 17.03 \mathrm{~g}(\mathrm{P}<0.01), 354.4 \pm 19.79 \mathrm{~g}(\mathrm{P}<0.01)$, $355.3 \pm 21.62 \mathrm{~g}(\mathrm{P}<0.01), 358.14 \pm 21.39 \mathrm{~g}(\mathrm{P}<0.05), 356 \pm 18.94 \mathrm{~g}$ $(\mathrm{P}<0.05), 358.88 \pm 19.26 \mathrm{~g}(\mathrm{P}<0.05)$, at $0,2,3,4,5,6$ weeks, respectively (Fig. 1A). After liraglutide treatment, the body weight of ZDF rats decreased to $357.33 \pm 13.31 \mathrm{~g}, 333.12 \pm 18.08 \mathrm{~g}$ $(\mathrm{P}<0.05), 332.2 \pm 12.57 \mathrm{~g}(\mathrm{P}<0.05), 333.2 \pm 6.22 \mathrm{~g}(\mathrm{P}<0.05)$, $334.5 \pm 13.21 \mathrm{~g}(\mathrm{P}<0.05), 335.5 \pm 13.13 \mathrm{~g}(\mathrm{P}<0.05)$ at each time point, respectively (Fig. 1A). Additionally, the fasting blood glucose level was much higher in ZDF rats $(16.28 \pm 4.0 \mathrm{mmol} / \mathrm{l}$ $(\mathrm{P}<0.001), 22.36 \pm 2.44 \mathrm{mmol} / \mathrm{l}(\mathrm{P}<0.001), 24.38 \pm 1.55 \mathrm{mmol} / \mathrm{l}$ $(\mathrm{P}<0.001), 26.08 \pm 1.67 \mathrm{mmol} / \mathrm{l}(\mathrm{P}<0.001), 24.26 \pm 1.49 \mathrm{mmol} / \mathrm{l}$ $(\mathrm{P}<0.001), 24.71 \pm 2.19 \mathrm{mmol} / 1(\mathrm{P}<0.001))$ than in $\mathrm{ZL}$ rats $(4.93 \pm 0.67,5.35 \pm 0.24,6.45 \pm 0.97,6.38 \pm 1.11,7.05 \pm 2.09$, $7.65 \pm 2.90 \mathrm{mmol} / \mathrm{l}$ ) at $0,2,3,4,5,6$ weeks, respectively (Fig. 1B). However, liraglutide treatment significantly decreased the fasting blood glucose in ZDF rats $(16.9 \pm 1.71 \mathrm{mmol} / 1$, $17.91 \pm 3.25 \mathrm{mmol} / \mathrm{l}(\mathrm{P}<0.05), 19.56 \pm 2.34 \mathrm{mmol} / \mathrm{l}(\mathrm{P}<0.01)$, $22.8 \pm 1.14 \mathrm{mmol} / \mathrm{l}(\mathrm{P}<0.05), 21.58 \pm 3.18 \mathrm{mmol} / \mathrm{l}(\mathrm{P}<0.05)$, $19.67 \pm 4.20 \mathrm{mmol} / \mathrm{l}(\mathrm{P}<0.05))$ at $0,2,3,4,5,6$ weeks, respectively (Fig. 1B). The serum levels of ALT $(181.3 \pm 95.8 \mathrm{U} / \mathrm{l})$ and AST $(178.4 \pm 56.7 \mathrm{U} / \mathrm{l})$ were significantly higher in ZDF rats than in $\mathrm{ZL}$ rats (ALT: $47.9 \pm 18.6 \mathrm{U} / 1(\mathrm{P}<0.01)$; AST: $107.9 \pm 20.8 \mathrm{U} / 1(\mathrm{P}<0.05))$, but the levels decreased after liraglutide treatment for six weeks (ALT: 86.5 $\pm 28.5 \mathrm{U} / 1(\mathrm{P}<0.05)$; AST: $100.5 \pm 22.0 \mathrm{U} / 1(\mathrm{P}<0.05))$ (Fig. $1 \mathrm{C}$ and D). Furthermore, the serum LDL-c $(3.57 \pm 0.57 \mathrm{mmol} / 1(\mathrm{P}<0.001))$ and $\mathrm{TC}$ levels $(6.3 \pm 1.1 \mathrm{mmol} / \mathrm{l}(\mathrm{P}<0.001))$ were significantly higher in $\mathrm{ZDF}$ rats than in the control (LDL-c: $0.55 \pm 0.24 \mathrm{mmol} / \mathrm{l}$; TC: $1.94 \pm 0.66 \mathrm{mmol} / \mathrm{l})$, and liraglutide decreased the serum LDL-c $(2.1 \pm 0.99 \mathrm{mmol} / \mathrm{l}(\mathrm{P}<0.05))$ and TC levels $(4.74 \pm 1.24 \mathrm{mmol} / \mathrm{l}$ $(\mathrm{P}<0.05))$ in $\mathrm{ZDF}$ rats (Fig. $1 \mathrm{E}$ and $\mathrm{F})$. These data indicated that liraglutide treatment improved metabolic disorders in ZDF rats.

Liraglutide decreases hepatic lipidandglycogen accumulation . $\mathrm{H} \& \mathrm{E}$ staining showed larger vacuoles in the livers of ZDF rats than in those of $\mathrm{ZL}$ rats, indicating the accumulation of lipid droplets (Fig. 2A). In contrast, liraglutide treatment reduced the appearance of large vacuoles in the livers of ZDF rats (Fig. 2A). The hepatic lipid contents were also higher in the livers of ZDF rats $(1.24 \pm 0.33 \mathrm{mg} / \mathrm{g}$ vs. $0.14 \pm 0.13 \mathrm{mg} / \mathrm{g}$, $\mathrm{P}<0.001)$, but this increase was significantly reduced after liraglutide treatment for 6 weeks $(0.80 \pm 0.09 \mathrm{mg} / \mathrm{g}, \mathrm{P}<0.05)$ (Fig. 2B). Interestingly, both PAS staining and glycogen quantification indicated the accumulation of glycogen in the livers of $\mathrm{ZDF}$ rats $(2.48 \pm 0.25 \mathrm{mg} / \mathrm{g})$ than those of $\mathrm{ZL}$ rats $(1.10 \pm 0.48 \mathrm{mg} / \mathrm{g})(\mathrm{P}<0.001)($ Fig. $2 \mathrm{C}$ and $\mathrm{D})$. We proposed that although glycogen synthesis was increased in ZDF rats, the level of glycogen synthesis was still much less than that needed to handle the input from blood glucose, thereby leading to a sustained high fasting blood glucose level. Not surprisingly, liraglutide therapy further led to an increase in the glycogen content of the livers of ZDF rats $(3.19 \pm 0.54 \mathrm{mg} / \mathrm{g}, \mathrm{P}<0.05)$ (Fig. 2C and D), indicating the protective role of liraglutide in the livers of diabetic rats.

Liraglutide reduces liver cell apoptosis in ZDF rats. Glucolipotoxicity-induced liver cell apoptosis has been widely reported $(20,21)$. Hence, we examined liver cell apoptosis in $\mathrm{ZDF}$ rats in the presence or absence of liraglutide. As shown in Fig. 3A, more apoptotic cells were found in the livers of ZDF rats than in those of ZL rats. However, after liraglutide therapy for 6 weeks, cell apoptosis was markedly reduced, as indicated by TUNEL staining (Fig. 3A). IHC staining also showed increased expression of c-caspase 3 in the livers of ZDF rats, while liraglutide treatment decreased the levels of c-caspase 3 (Fig. 3B). Similarly, Western blot showed that c-caspase3 expression was significantly higher in the livers of ZDF rats $(2.29 \pm 0.36)$ than in those of ZL rats $(1 \pm 0.17)(\mathrm{P}<0.01)$ but that this increase could largely be reversed by liraglutide treatment $(1.43 \pm 0.20)(\mathrm{P}<0.05$; Fig. 3C).

Liraglutide increases the activity of antioxidant enzymes. MDA, a lipid peroxidation product, was quantified in the serum and livers of ZL and ZDF rats. Compared with ZL rats (3.3 $\pm 0.7 \mathrm{ZD} \mathrm{ng} / \mathrm{ml}), \mathrm{ZDF}$ rats tended to have higher MDA content $(3.8 \pm 1.2 \mathrm{ng} / \mathrm{ml})$, but the increase was not significant (Fig. 4A). In the livers of ZDF rats, enhancement of MDA was found $(2.26 \pm 0.34 \mathrm{ng} / \mathrm{mg}$ protein vs. $1.65 \pm 0.29 \mathrm{ng} / \mathrm{mg}$ protein), but no statistical significance (Fig. 4D). After liraglutide 

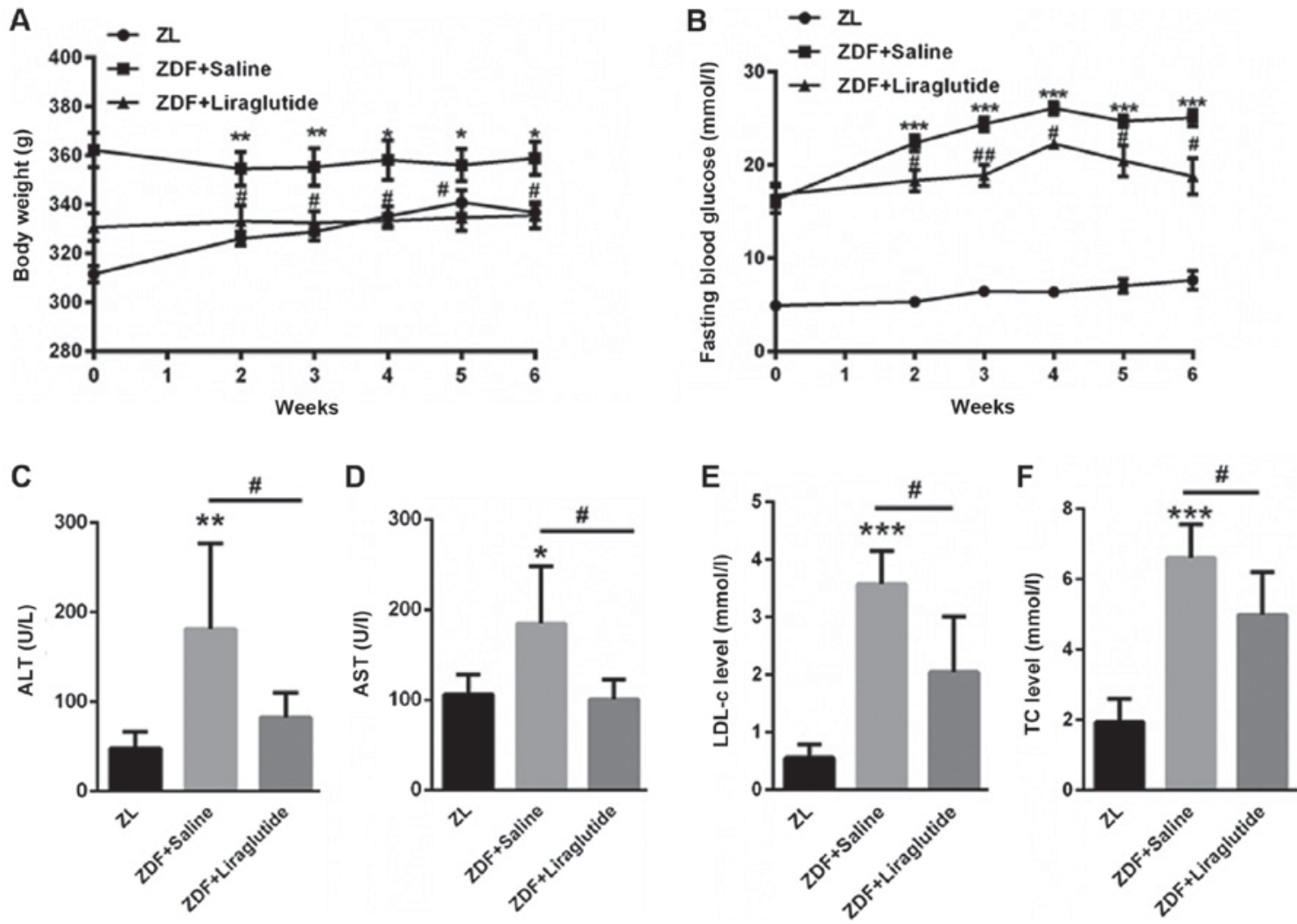

Figure 1. Liraglutide treatment ameliorates metabolic disorders in ZDF rats. (A) Body weight and (B) fasting blood glucose were evaluated in ZL rats and ZDF rats with or without liraglutide treatment. Serum levels of (C) ALT and (D) AST were determined in ZL rats and ZDF rats with or without liraglutide treatment. Serum (E) LDL-c and (F) TC levels were examined in ZL rats and ZDF rats with or without liraglutide treatment. Data are presented as the mean \pm standard error ( $\mathrm{n}=5$ rats for each group). ${ }^{\mathrm{P}} \mathrm{P}<0.05,{ }^{* *} \mathrm{P}<0.01$ and ${ }^{* * *} \mathrm{P}<0.001$ vs. $\mathrm{ZL} ;{ }^{*} \mathrm{P}<0.05$ and ${ }^{\# *} \mathrm{P}<0.01$ vs. ZDF+Saline. ZDF, Zucker diabetic fatty; ZL, Zucker lean; ALT, alanine aminotransferase; AST, aspartate aminotransferase; LDL-c, low density lipoprotein cholesterol; TC, total cholesterol.

A

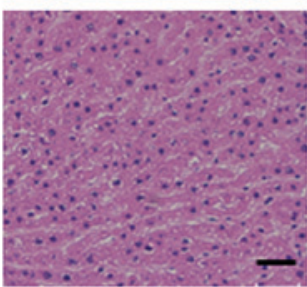

ZL rats

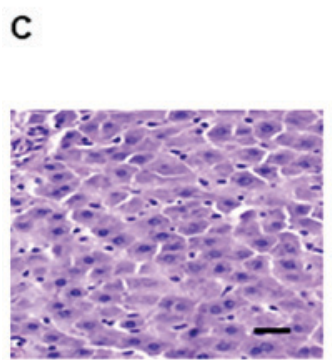

$\mathrm{ZL}$ rats

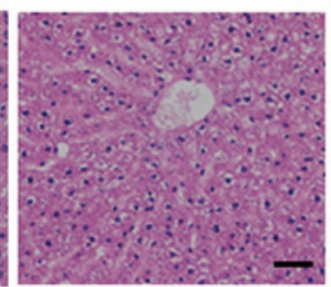

ZDF rats

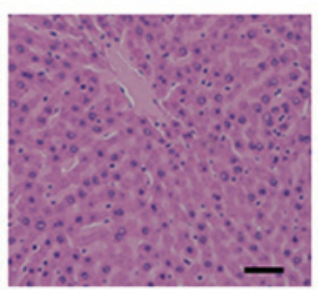

ZDF rats+Liralutide

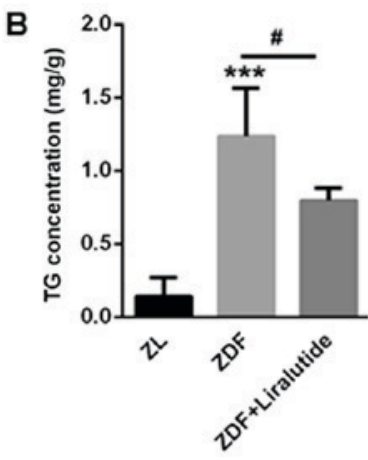

D

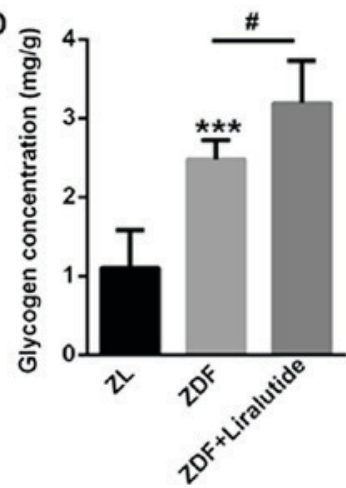

Figure 2. Liraglutide decreases hepatic lipid and glycogen accumulation. (A) Hematoxylin and eosin staining revealed that liraglutide treatment reduced the appearance of large vacuoles in the livers of ZDF rats. Scale bars, $50 \mu \mathrm{m}$. (B) Quantification of lipid content in the livers of ZL and ZDF rats with or without liraglutide therapy. (C) Periodic Acid Schiff staining revealed that liraglutide decreased glycogen accumulation in the livers of ZDF rats. Scale bars, $50 \mu \mathrm{m}$. (D) Quantification of glycogen content in the livers of ZL and ZDF rats with or without liraglutide therapy. Data are presented as the mean \pm standard error ( $\mathrm{n}=5$ rats for each group). ${ }^{* * *} \mathrm{P}<0.001 \mathrm{vs.} \mathrm{ZL}$ rats; ${ }^{\#} \mathrm{P}<0.05$, as indicated. ZDF, Zucker diabetic fatty; ZL, Zucker lean. 

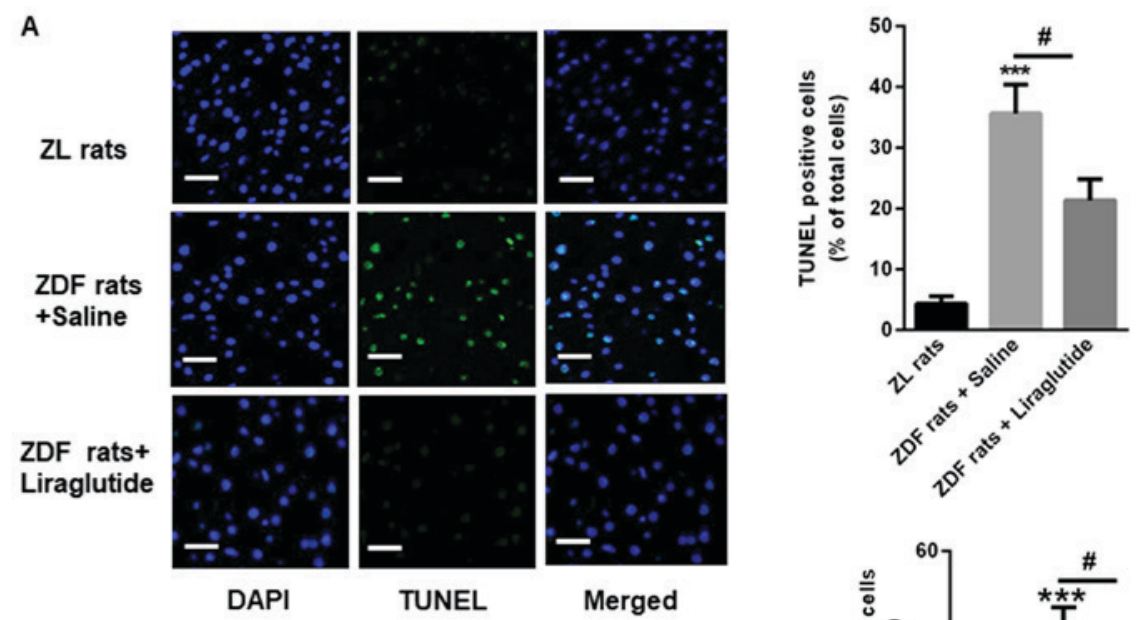

B
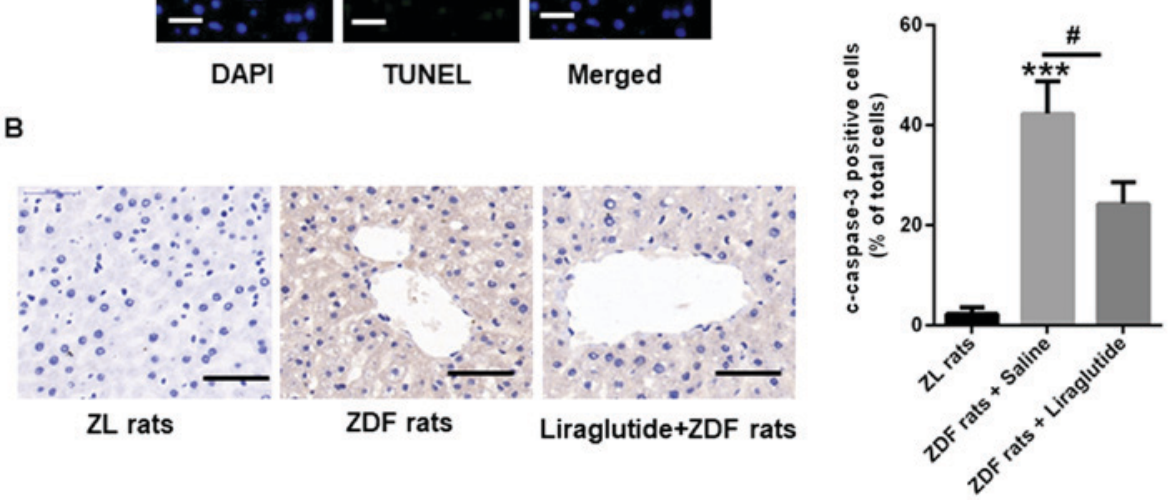

C

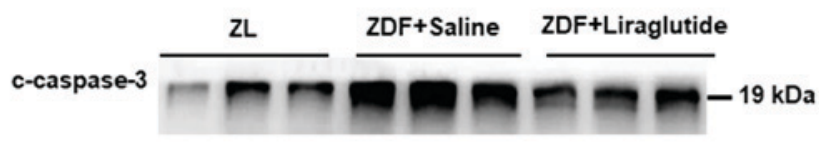

$\beta$-actin
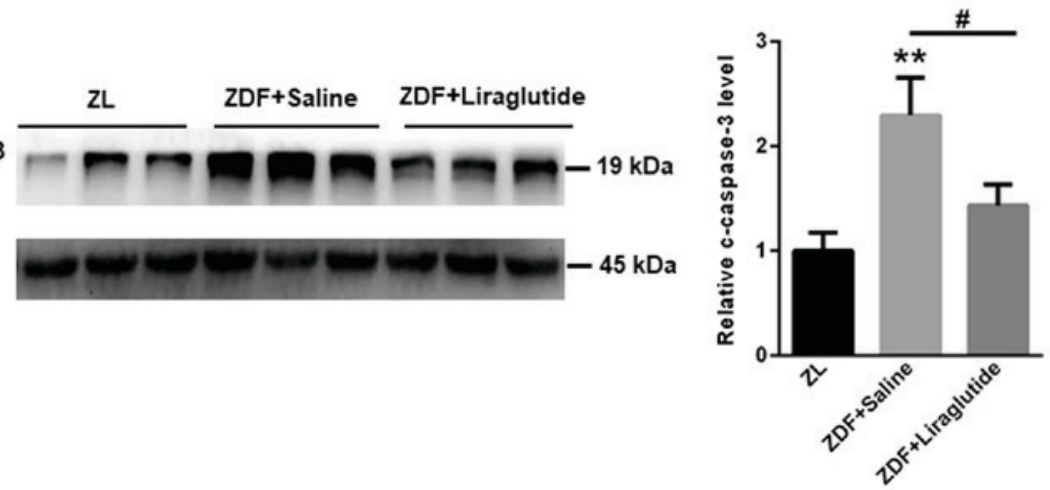

Figure 3. Liraglutide reduces liver cell apoptosis in ZDF rats. (A) Terminal deoxynucleotidyl-transferase-mediated dUTP nick end labeling staining for liver cell apoptosis in ZDF rats in the presence or absence of liraglutide. Scale bars, $50 \mu \mathrm{m}$. (B) Immunohistochemical staining for c-caspase3 in ZDF rats in the presence or absence of liraglutide. Scale bars, $50 \mu \mathrm{m}$. (C) Western blot analysis revealed that c-caspase 3 expression was significantly higher in the livers of ZDF rats than in those of ZL rats; however, this increase was reversed by liraglutide treatment. Data are presented as the mean \pm standard error $(\mathrm{n}=5$ rats for each group). ${ }^{* * *} \mathrm{P}<0.01$ and ${ }^{* * *} \mathrm{P}<0.001$ vs. ZL rats; ${ }^{*} \mathrm{P}<0.05$, as indicated. ZDF, Zucker diabetic fatty; ZL, Zucker lean; c-caspase3, cleaved caspase 3 .

treatment for 6 weeks, the serum and hepatic MDA contents both decreased to $1.2 \pm 0.5 \mathrm{ng} / \mathrm{ml}(\mathrm{P}<0.05)$ and $1.92 \pm 0.12 \mathrm{ng} / \mathrm{mg}$ protein $(\mathrm{P}<0.05)$ (Fig. 4A and D). Moreover, the activity of $\mathrm{GSH}$ and SOD was lower in the serum (GSH: $2.2 \pm 0.1 \mathrm{U} / \mathrm{ml}$ $(\mathrm{P}>0.05)$; SOD: $125.3 \pm 6.2 \mathrm{U} / \mathrm{ml}(\mathrm{P}<0.05))$ and livers $(\mathrm{GSH}$ : $84.3 \pm 4.9 \mathrm{ng} / \mathrm{mg}$ protein $(\mathrm{P}>0.05)$; SOD: $374.64 \pm 0.48 \mathrm{ng} / \mathrm{mg}$ protein $(\mathrm{P}<0.05))$ of $\mathrm{ZDF}$ rats than those in the serum (GSH: $2.4 \pm 0.1 \mathrm{U} / \mathrm{ml}$; SOD: $137.4 \pm 5.2 \mathrm{U} / \mathrm{ml}$ ) and livers $(\mathrm{GSH}$ : $137.7 \pm 46.1 \mathrm{ng} / \mathrm{mg}$ protein; SOD: $433.1 \pm 15.1 \mathrm{ng} / \mathrm{mg}$ protein) of $\mathrm{ZL}$ rats (Fig. $4 \mathrm{~B}, \mathrm{C}, \mathrm{E}$, and $\mathrm{F}$ ). In addition, liraglutide treatment significantly increased the activity of these antioxidant enzymes in both the serum (GSH: $2.5 \pm 0.1 \mathrm{U} / \mathrm{ml}(\mathrm{P}<0.05)$; SOD: 142.1 $\pm 6.2 \mathrm{U} / \mathrm{ml}(\mathrm{P}<0.05))$ and livers (GSH: $98.3 \pm 5.8 \mathrm{ng} / \mathrm{mg}$ protein $(\mathrm{P}<0.05)$; SOD: $414.8 \pm 17.0 \mathrm{ng} / \mathrm{mg}$ protein $(\mathrm{P}<0.05))$ of $\mathrm{ZDF}$ rats (Fig. 4B, C, E, and F). These data showed that liraglutide could enhance the antioxidant capacity of ZDF rats.
Liraglutide enhances the activation of the NRF2 signaling pathway. NRF2/HO-1 is involved in an important antioxidant signaling pathway that participates in the lipid peroxidation found in hepatic metabolic disorders. Hence, the expression of NRF2 and downstream antioxidant enzymes, including NQO1 and HO-1, was explored. As shown in Fig. 5, no significant changes in NRF2 and NQO1 were found in the livers of $\mathrm{ZDF}$ rats compared with those of $\mathrm{ZL}$ rats $(\mathrm{P}>0.05)$. However, the expression of HO-1 was higher in the livers of ZDF rats $(\mathrm{P}<0.05)$, which may be due to a stress-induced self-regulatory mechanism. Strikingly, the expression of NRF2 $(\mathrm{P}<0.05)$, NQO1 $(\mathrm{P}<0.05)$ and HO-1 $(\mathrm{P}<0.05)$ was significantly upregulated in the livers of $\mathrm{ZDF}$ rats after liraglutide treatment (Fig. 5). These data indicated that the activation of NRF2 signaling contributed to the improved antioxidant effects of liraglutide in the livers of ZDF rats. 

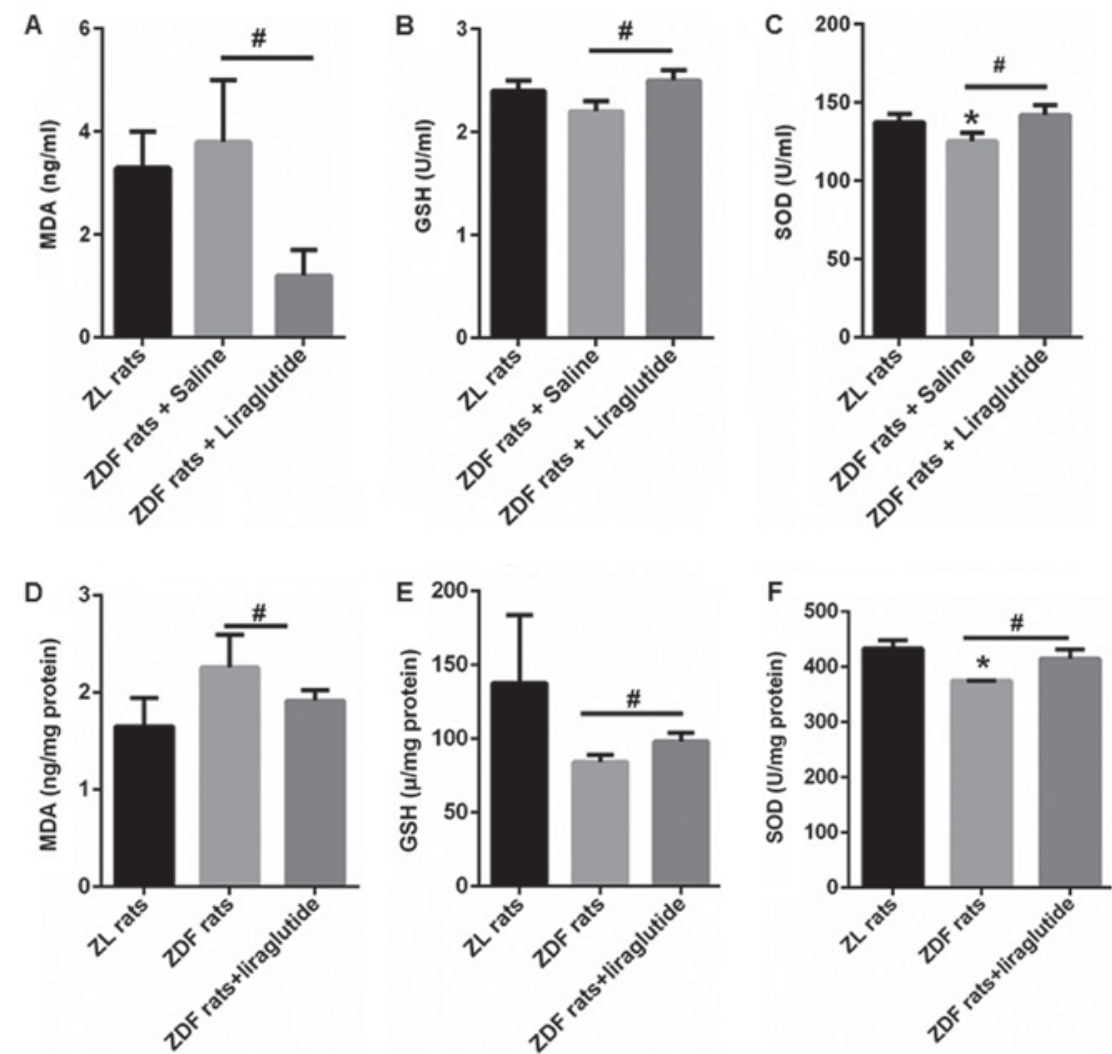

Figure 4. Liraglutide increases the activity of antioxidant enzymes. The (A) contents of serum MDA, (B) activity of serum GSH, (C) activity of serum SOD, (D) contents of hepatic MDA, (E) activity of hepatic GSH and (F) activity of hepatic SOD was determined in the ZL and ZDF rats with or without liraglutide treatment. Data are presented as the mean \pm standard error $\left(\mathrm{n}=5\right.$ rats for each group). ${ }^{*} \mathrm{P}<0.05 \mathrm{vs}$. $\mathrm{ZL}$ rats; ${ }^{\prime \prime} \mathrm{P}<0.05$, as indicated. ZDF, Zucker diabetic fatty; ZL, Zucker lean; MDA, malondialdehyde; SOD, superoxide dismutase; GSH, glutathione peroxidase.
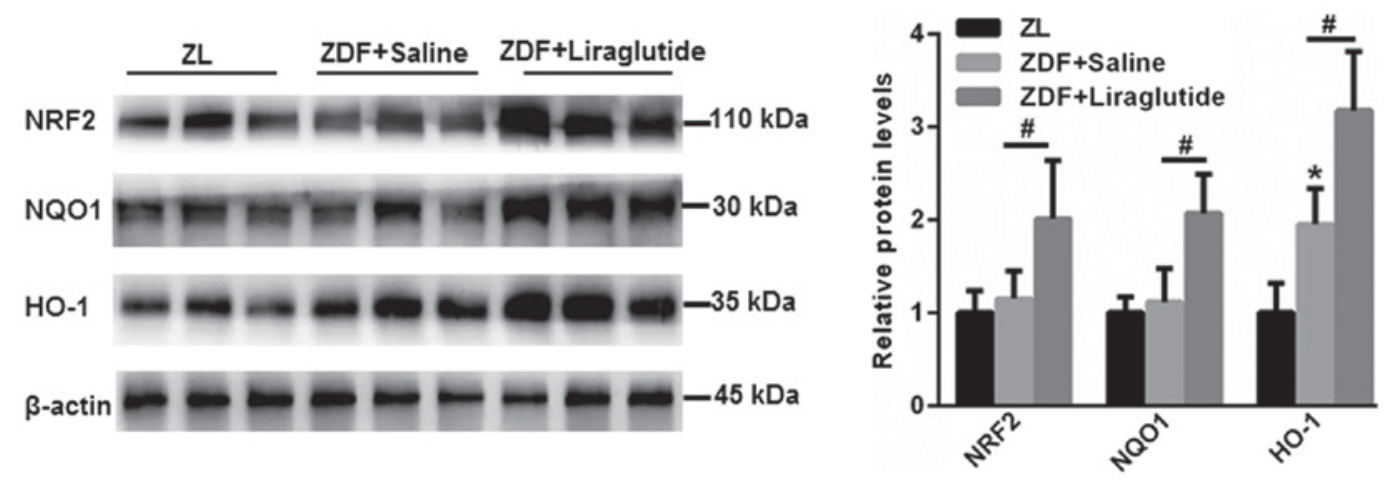

Figure 5. Western blot assay revealed that liraglutide enhanced the expression of NRF2, NQO1 and HO-1. Data are presented as the mean \pm standard error ( $\mathrm{n}=5$ rats for each group). ${ }^{*} \mathrm{P}<0.05 \mathrm{vs.} \mathrm{ZL}$ rats; ${ }^{\#} \mathrm{P}<0.05$, as indicated. $\mathrm{ZDF}$, Zucker diabetic fatty; ZL, Zucker lean; NRF2, nuclear factor-erythroid 2-related factor 2; NQO1, nicotinamide adenine dinucleotide phosphate quinone dehydrogenase 1; HO-1, heme oxygenase-1.

\section{Discussion}

Metabolic disorders have become an alarming public health trend around the world, and they result in an increased risk of developing cardiovascular disease, type 2 diabetes and NAFLD, which are referred to as metabolic syndromes (22-24). Liraglutide is a GLP-1 analog that shares 97\% sequence identity with human GLP-1 (16). In January 2010, liraglutide was approved by the FDA for the treatment of hyperglycemia in patients with T2DM. Subsequently, an increasing number of studies have shown the protective role of liraglutide in the livers of diabetic and obesity patients $(25,26)$.
High glucose and lipid accumulation can trigger ROS generation and cell apoptosis, induce inflammation, and finally results in the pathogenesis of liver disease (27). Here, we found that the fasting blood glucose, as well as lipid contents, AST, ALT, LDL-c, TC and the apoptosis index were elevated in the livers of ZDF rats than those of ZL rats. Therefore, we investigated whether liraglutide could protect liver cells against oxidative stress in ZDF rats. In line with previous studies $(28,29)$, our data showed that liraglutide significantly decreased the body weight, hyperglycemia and hyperlipidemia in ZDF rats compared with those in $\mathrm{ZL}$ rats. Furthermore, reduced liver cell apoptosis was observed in 
ZDF rats after liraglutide therapy for 6 weeks. These data validated the beneficial results of liraglutide in diabetic and obese ZDF rats.

The liver is characterized by high metabolic activity, and it is the major organ responsible for the biotransformation and subsequent detoxification of xenobiotics (30). Hence, the liver is at high risk of increased ROS and electrophile production, especially during the progression of diabetes, NAFLD, and other chronic liver diseases $(5,30,31)$. In hepatocytes, multiple antioxidant enzymes are dedicated to eliminating the endogenous and exogenous oxidants that result from lipid peroxidation (32). Among them, many enzymes are transcribed from genes, including antioxidant response elements (AREs) in their promoter regions (33). NRF2 is well known to induce the expression of ARE-containing genes encoding antioxidant enzymes in response to cellular stresses, including ROS (34). Compared with wild-type mice, $\mathrm{NRF} 2^{-/}$mice are more susceptible to chemical-induced oxidative/electrophilic stress in the liver (35,36). Additionally, 2,3,7,8-tetrachlorodibenzo-p-dioxin (TCDD)-induced oxidative damage can be largely improved by overexpression of NRF2 in mice (37). Furthermore, knockout of NRF2 in hepatocytes leads to increased liver cell injury under the conditions of excessive iron accumulation (38). Hence, NRF2 activity plays a key role in the protection against oxidative stress-induced liver cell injury.

Liraglutide was shown to alleviate oxidative stress in $\beta$-islet cells or in the brains of diabetic mice subjected to middle cerebral artery occlusion $(39,40)$. However, whether liraglutide protects livers from lipid peroxidation via the NRF2 signaling pathway is unclear. For the first time, we show novel data indicating that the expression of the antioxidant transcription factor NRF2, as well as the transcription of downstream target genes, including NQO1 and HO-1, was increased by liraglutide treatment. Additionally, both serum and hepatic GSH and SOD levels were enhanced after liraglutide therapy. This change ultimately leads to a lower state of oxidative stress in liver cells, thereby improving oxidative stress-induced liver cell injury. Multiple studies have suggested that ROS markedly enhance the transformation of fatty liver into non-alcoholic steatohepatitis (NASH) $(41,42)$. Excessive ROS generated by the overconsumption of lipid and glucose results in burden on quenching by hepatic antioxidants (42). NRF2 plays a key role in regulating multiple antioxidant-related genes that are associated in the cellular defense against oxidative stress (43). It is well suggested that enhanced antioxidant activity improves liver cell apoptosis via NRF2 signaling $(44,45)$. For instance, safflower yellow B is demonstrated to inhibit HepG2 cell apoptosis induced by oxidative stress through activating the AKT/Nrf2 pathway (44). In the present study, we showed liraglutide protects liver cells from hepatic glucolipotoxicity-induced oxidative stress and liver cell apoptosis in ZDF rats through NRF2-dependent mechanism.

Even though, whether the effects of liraglutide on hepatocytes apoptosis are direct effects or not via NRF2 signaling remains to be further elucidated. GLP-1 is demonstrated to be beneficial against cell apoptosis in various circumstances. However, the presence of GLP-1 receptor in hepatocytes is controversial. Recently, it is indicated that the metabolic products of GLP-1 retain important antioxidant and anti-apoptotic activities that are GLP-1 R independent (46). For instance, GLP-1 has been shown to protect endothelial cells from advanced glycation end products (AGEs)-induced apoptosis by inhibiting the release of mitochondrion cytochrome c (47). And GLP-1 analogue, liraglutide, may prevent high glucose induced mitochondrial fragmentation and apoptosis in human endothelial cells via inducing mitochondrial fusion processes (48). Additionally, liraglutide is also suggested to protect cardiomyocyte from oxidative stress and apoptosis via activating AMPK-Sirt1 pathway (49). In neural tissues, the beneficial effects of GLP-1 on cell apoptosis is indicated to be due to the activation of the Akt pathway (50). Based on these findings, we propose that the anti-apoptotic effects of GLP-1 may be NRF-2 dependent and independent of GLP-1R in liver tissues.

In summary, liraglutide can enhance the antioxidant activity of liver cells by activating the NRF2 signaling pathway, which then results in a decrease in liver cell apoptosis induced by glucolipotoxicity in ZDF rats.

\section{Acknowledgements}

Not applicable.

\section{Funding}

The present study was supported by grants (grant nos. 81570789 and 81700765) from National Natural Science Foundation of China.

\section{Availability of data and materials}

The datasets used and/or analyzed during the current study are available from the corresponding author on reasonable request.

\section{Authors' contributions}

JG and CL performed the experiments and analyzed the data. CY and BL performed the animal experiments. JW, YL and PY performed part of the animal experiments. GH and JL designed the experiments, analyzed the data and gave final approval of the version to be published. All authors read and approved the final manuscript.

\section{Ethics approval and consent to participate}

The present study was approved by the Animal Ethics Committee at the MOH Key Laboratory of Geriatrics, Beijing Hospital (no. BJHMOH-2015-1002).

\section{Consent for publication}

Not applicable.

\section{Competing interests}

The authors declare that they have no competing interests. 


\section{References}

1. Castro AV, Kolka CM, Kim SP and Bergman RN: Obesity, insulin resistance and comorbidities? Mechanisms of association. Arq Bras Endocrinol Metabol 58: 600-609, 2014

2. Li Z, Xue J, Chen P, Chen L, Yan S and Liu L: Prevalence of nonalcoholic fatty liver disease in mainland of China: A meta-analysis of published studies. J Gastroenterol Hepatol 29 42-51, 2014.

3. Xu Y, Wang L, He J, Bi Y, Li M, Wang T, Wang L, Jiang Y, Dai M, Lu J, et al: Prevalence and control of diabetes in Chinese adults. JAMA 310: 948-959, 2013.

4. Yang W, Lu J, Weng J, Jia W, Ji L, Xiao J, Shan Z, Liu J, Tian H, Ji Q, et al: Prevalence of diabetes among men and women in China. N Engl J Med 362: 1090-1101, 2010

5. Serviddio G, Bellanti F and Vendemiale G: Free radical biology for medicine: Learning from nonalcoholic fatty liver disease. Free Radic Biol Med 65: 952-968, 2013

6. Stein Y and Shapiro B: Uptake and metabolism of triglycerides by the rat liver. J Lipid Res 1: 326-331, 1960.

7. Mota M, Banini BA, Cazanave SC and Sanyal AJ: Molecular mechanisms of lipotoxicity and glucotoxicity in nonalcoholic fatty liver disease. Metabolism 65: 1049-1061, 2016

8. Zámbó V, Simon-Szabó L, Szelényi P, Kereszturi E, Bánhegyi G and Csala M: Lipotoxicity in the liver. World J Hepatol 5: 550-557, 2013

9. Hine CM and Mitchell JR: NRF2 and the phase II response in acute stress resistance induced by dietary restriction. J Clin Exp Pathol S4: pii: 7329, 2012.

10. Jaiswal AK: Nrf2 signaling in coordinated activation of antioxidant gene expression. Free Radic Biol Med 36: 1199-1207, 2004

11. Schaedler S, Krause J, Himmelsbach K, Carvajal-Yepes M, Lieder F, Klingel K, Nassal M, Weiss TS, Werner S and Hildt E: Hepatitis B virus induces expression of antioxidant response element-regulated genes by activation of Nrf2. J Biol Chem 285 41074-41086, 2010.

12. Holland R and Fishbein JC: Chemistry of the cysteine sensors in Kelch-like ECH-associated protein 1. Antioxid Redox Signal 13: 1749-1761, 2010

13. Volonte D, Liu Z, Musille PM, Stoppani E, Wakabayashi N, Di YP, Lisanti MP, Kensler TW and Galbiati F: Inhibition of nuclear factor-erythroid 2-related factor (Nrf2) by caveolin-1 promotes stress-induced premature senescence. Mol Biol Cell 24: $1852-1862,2013$

14. Nauck MA: Incretin-based therapies for type 2 diabetes mellitus: Properties, functions, and clinical implications. Am J Med 124 (1 Suppl): S3-S18, 2011.

15. Kazafeos K: Incretin effect: GLP-1, GIP, DPP4. Diabetes Res Clin Pract 93 (Suppl 1): S32-S36, 2011.

16. Zueger PM, Schultz NM and Lee TA: Cost effectiveness of liraglutide in type II diabetes: A systematic review. Pharmacoeconomics 32: 1079-1091, 2014.

17. de Mesquita FC, Guixé-Muntet S, Fernández-Iglesias A, Maeso-Díaz R, Vila S, Hide D, Ortega-Ribera M, Rosa JL, García-Pagán JC, Bosch J, et al: Liraglutide improves liver microvascular dysfunction in cirrhosis: Evidence from translational studies. Sci Rep 7: 3255, 2017.

18. Liang Y, Li Z, Liang S, Li Y, Yang L, Lu M, Gu HF and Xia N: Hepatic adenylate cyclase 3 is upregulated by Liraglutide and subsequently plays a protective role in insulin resistance and obesity. Nutr Diabetes 6: e191, 2016.

19. Kleiner DE, Brunt EM, Van Natta M, Behling C, Contos MJ, Cummings OW, Ferrell LD, Liu YC, Torbenson MS, Unalp-Arida A, et al: Design and validation of a histological scoring system for nonalcoholic fatty liver disease. Hepatology 41 $1313-1321,2005$

20. Kowluru A and Matti A: Hyperactivation of protein phosphatase 2A in models of glucolipotoxicity and diabetes: Potential mechanisms and functional consequences. Biochem Pharmacol 84: 591-597, 2012

21. Purwana I, Liu JJ, Portha B and Buteau J: HSF1 acetylation decreases its transcriptional activity and enhances glucolipotoxicity-induced apoptosis in rat and human beta cells Diabetologia 60: 1432-1441, 2017.

22. Ford ES, Giles WH and Dietz WH: Prevalence of the metabolic syndrome among US adults: Findings from the third National Health and Nutrition Examination Survey. JAMA 287: 356-359, 2002 .
23. Boudreau DM, Malone DC, Raebel MA, Fishman PA, Nichols GA, Feldstein AC, Boscoe AN, Ben-Joseph RH, Magid DJ and Okamoto LJ: Health care utilization and costs by metabolic syndrome risk factors. Metab Syndr Relat Disord 7: 305-314, 2009.

24. Alberti KG, Zimmet P and Shaw J: Metabolic syndrome-a new world-wide definition. A consensus statement from the International diabetes federation. Diabet Med 23: 469-480, 2006

25. Polyzos SA, Kountouras J and Mantzoros CS: Adipose tissue, obesity and non-alcoholic fatty liver disease. Minerva Endocrinol 42: 92-108, 2017.

26. Cuthbertson DJ, Irwin A, Gardner CJ, Daousi C, Purewal T, Furlong N, Goenka N, Thomas EL, Adams VL, Pushpakom SP, et al: Improved glycaemia correlates with liver fat reduction in obese, type 2 diabetes, patients given glucagon-like peptide-1 (GLP-1) receptor agonists. PLoS One 7: e50117, 2012.

27. Li Q, Lin Y, Wang S, Zhang L and Guo L: GLP-1 inhibits high-glucose-induced oxidative injury of vascular endothelial cells. Sci Rep 7: 8008, 2017.

28. Brand CL, Galsgaard ED, Tornehave D, Rømer J, Gotfredsen CF, Wassermann K, Knudsen LB, Vølund A and Sturis J: Synergistic effect of the human GLP-1 analogue liraglutide and a dual PPARalpha/gamma agonist on glycaemic control in Zucker diabetic fatty rats. Diabetes Obes Metab 11: 795-803, 2009.

29. Larsen PJ, Wulff EM, Gotfredsen CF, Brand CL, Sturis J, Vrang N, Knudsen LB and Lykkegaard K: Combination of the insulin sensitizer, pioglitazone, and the long-acting GLP-1 human analog, liraglutide, exerts potent synergistic glucose-lowering efficacy in severely diabetic ZDF rats. Diabetes Obes Metab 10 301-311, 2008

30. Zeng J, Deng S, Wang Y, Li P, Tang L and Pang Y: Specific inhibition of Acyl-CoA oxidase-1 by an acetylenic acid improves hepatic lipid and reactive oxygen species (ROS) metabolism in rats fed a high fat diet. J Biol Chem 292: 3800-3809, 2017.

31. Levonen AL, Hill BG, Kansanen E, Zhang J and Darley-Usmar VM: Redox regulation of antioxidants, autophagy, and the response to stress: Implications for electrophile therapeutics. Free Radic Biol Med 71: 196-207, 2014.

32. Rushmore TH, Morton MR and Pickett CB: The antioxidant responsive element. Activation by oxidative stress and identification of the DNA consensus sequence required for functional activity. J Biol Chem 266: 11632-11639, 1991.

33. Lee JM, Moehlenkamp JD, Hanson JM and Johnson JA Nrf2-dependent activation of the antioxidant responsive element by tert-butylhydroquinone is independent of oxidative stress in IMR-32 human neuroblastoma cells. Biochem Biophys Res Commun 280: 286-292, 2001.

34. Lee JM, Calkins MJ, Chan K, Kan YW and Johnson JA Identification of the NF-E2-related factor-2-dependent genes conferring protection against oxidative stress in primary cortical astrocytes using oligonucleotide microarray analysis. J Biol Chem 278: 12029-12038, 2003.

35. Klaassen CD and Reisman SA: Nrf2 the rescue: Effects of the antioxidative/electrophilic response on the liver. Toxicol Appl Pharmacol 244: 57-65, 2010.

36. Liu J, Wu KC, Lu YF, Ekuase E and Klaassen CD: Nrf2 protection against liver injury produced by various hepatotoxicants. Oxid Med Cell Longev 2013: 305861, 2013

37. Lu H, Cui W and Klaassen CD: Nrf2 protects against 2,3,7,8-tetrachlorodibenzo-p-dioxin (TCDD)-induced oxidative injury and steatohepatitis. Toxicol Appl Pharmacol 256: 122-135, 2011.

38. Silva-Gomes S, Santos AG, Caldas C, Silva CM, Neves JV, Lopes J, Carneiro F, Rodrigues PN and Duarte TL: Transcription factor NRF2 protects mice against dietary iron-induced liver injury by preventing hepatocytic cell death. J Hepatol 60: 354-361, 2014

39. Li PC, Liu LF, Jou MJ and Wang HK: The GLP-1 receptor agonists exendin- 4 and liraglutide alleviate oxidative stress and cognitive and micturition deficits induced by middle cerebral artery occlusion in diabetic mice. BMC Neurosci 17: 37, 2016.

40. Shimoda M, Kanda $\mathrm{Y}$, Hamamoto S, Tawaramoto $\mathrm{K}$, Hashiramoto $\mathbf{M}$, Matsuki $\mathbf{M}$ and Kaku K: The human glucagon-like peptide-1 analogue liraglutide preserves pancreatic beta cells via regulation of cell kinetics and suppression of oxidative and endoplasmic reticulum stress in a mouse model of diabetes. Diabetologia 54: 1098-1108, 2011. 
41. He W, Xu Y, Zhang C, Lu J, Li J, Xiang D, Yang J, Chang M and Liu D: Hepatoprotective effect of calculus bovis sativus on nonalcoholic fatty liver disease in mice by inhibiting oxidative stress and apoptosis of hepatocytes. Drug Des Devel Ther 11: 3449-3460, 2017.

42. Lim JS, Mietus-Snyder M, Valente A, Schwarz JM and Lustig RH: The role of fructose in the pathogenesis of NAFLD and the metabolic syndrome. Nat Rev Gastroenterol Hepatol 7: 251-264, 2010.

43. Musso G, Cassader M and Gambino R: Non-alcoholic steatohepatitis: Emerging molecular targets and therapeutic strategies. Nat Rev Drug Discov 15: 249-274, 2016.

44. Ma Z, Li C, Qiao Y, Lu C, Li J, Song W, Sun J, Zhai X, Niu J, Ren Q and Wen A: Safflower yellow B suppresses HepG2 cell injury induced by oxidative stress through the AKT/Nrf2 pathway. Int J Mol Med 37: 603-612, 2016.

45. Gokila Vani M, Kumar KJ, Liao JW, Chien SC, Mau JL, Chiang SS, Lin CC, Kuo YH and Wang SY: Antcin C from antrodia cinnamomea protects liver cells against free radical-induced oxidative stress and apoptosis in vitro and in vivo through Nrf2-dependent mechanism. Evid Based Complement Alternat Med 2013: 296082, 2013.

46. Thomas MC: The potential and pitfalls of GLP-1 receptor agonists for renal protection in type 2 diabetes. Diabetes Metab 43 (Suppl 1): 2S20-2S27, 2017.
47. Zhan Y, Sun HL, Chen H, Zhang H, Sun J, Zhang Z and Cai DH: Glucagon-like peptide-1 (GLP-1) protects vascular endothelial cells against advanced glycation end products (AGEs)-induced apoptosis. Med Sci Monit 18: BR286-BR291, 2012.

48. Schisano B, Harte AL, Lois K, Saravanan P, Al-Daghri N, Al-Attas O, Knudsen LB, McTernan PG, Ceriello A and Tripathi G: GLP-1 analogue, Liraglutide protects human umbilical vein endothelial cells against high glucose induced endoplasmic reticulum stress. Regul Pept 174: 46-52, 2012.

49. Inoue $\mathrm{T}$, Inoguchi $\mathrm{T}$, Sonoda $\mathrm{N}$, Hendarto $\mathrm{H}$, Makimura $\mathrm{H}$, Sasaki S, Yokomizo H, Fujimura Y, Miura D and Takayanagi R: GLP-1 analog liraglutide protects against cardiac steatosis, oxidative stress and apoptosis in streptozotocin-induced diabetic rats. Atherosclerosis 240: 250-259, 2015.

50. Kimura R, Okouchi M, Fujioka H, Ichiyanagi A, Ryuge F, Mizuno T, Imaeda K, Okayama N, Kamiya Y, Asai K and Joh T: Glucagon-like peptide-1 (GLP-1) protects against methylglyoxal-induced PC12 cell apoptosis through the PI3K/Akt/mTOR/GCLc/redox signaling pathway. Neuroscience 162: 1212-1219, 2009.

This work is licensed under a Creative Commons Attribution-NonCommercial-NoDerivatives 4.0 International (CC BY-NC-ND 4.0) License. 